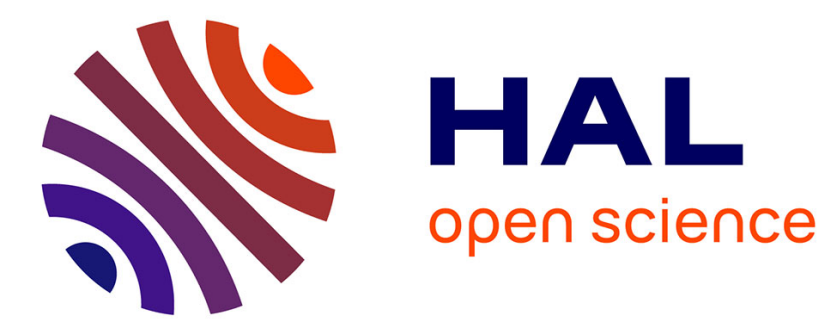

\title{
Chen Yun 1949-1956. Retouches à un portrait Thierry Pairault
}

\section{To cite this version:}

Thierry Pairault. Chen Yun 1949-1956. Retouches à un portrait. Études Chinoises, 1987, 6 (1), pp.59-93. halshs-00076576

\section{HAL Id: halshs-00076576 https://shs.hal.science/halshs-00076576}

Submitted on 25 May 2006

HAL is a multi-disciplinary open access archive for the deposit and dissemination of scientific research documents, whether they are published or not. The documents may come from teaching and research institutions in France or abroad, or from public or private research centers.
L'archive ouverte pluridisciplinaire HAL, est destinée au dépôt et à la diffusion de documents scientifiques de niveau recherche, publiés ou non, émanant des établissements d'enseignement et de recherche français ou étrangers, des laboratoires publics ou privés. 


\section{CHEN YUN 1949-1956 \\ Retouches à un portrait}

\section{Thierry Pairault}

Né en 1899 dans la province du Jiangsu, Liao Yuncheng dit Chen Yun entre très jeune aux Presses Commerciales (Shangwu yinshuguan) de Shanghai comme apprenti avant de devenir typographe. A ce titre il est un des rares vieux responsables du PCC qui puisse revendiquer une origine ouvrière. Entré au Parti en 1924, il devient l'un des membres du Bureau politique dès 1931. Après un séjour à Moscou de deux ans (1935-1937), il rejoint Yan'an et acquiert une réputation d'organisateur et de bon gestionnaire, principalement par ses activités dans la région du Shaan-Gan-Ning d'abord, puis en Mandchourie de 1945 à 1949. Dès lors, il se consacre à la réorganisation et au développement de l'économie chinoise.

Au cours des trente-sept années qui vont de 1949 à nos jours, l'activité de Chen Yun peut être divisée en trois périodes : 1949-1956; 1957-1978; 1979 à nos jours. La seconde période est certainement la moins intéressante pour la connaissance du personnage : au cours de ces vingt années Chen Yun vit dans une semi-disgrâce (il n'est pas purgé lors de la Révolution culturelle), et son activité est aussi difficile à saisir qu'à vérifier. Quant à la troisième période - la période actuelle -, elle pose plus de questions qu'elle ne permet d'en résoudre. Certes, Chen Yun fait une rentrée politique fracassante (peutêtre trop fracassante) en décembre 1978 lors du 3e plenum du Comité central; mais à peine rappelée par Deng Xiaoping, cette vivante incarnation du réalisme économique est rapidement écartée de la sphère où se décident

Études chinoises, vol. VI, $\mathrm{n}^{\circ} 1$ (1987) 
réformes et stratégies, pour se retrouver cantonnée dans le rôle éminemment honorable, mais d'une influence immédiate fort restreinte, de gardien de la vertu socialiste et de la discipline économique. Utilisé par Deng Xiaoping comme garant de son réalisme et de ses intentions réformistes, puis rejeté, une fois la virginité politique de Deng retrouvée, Chen Yun n'a-t-il pas été alors récupéré par une tendance "réformiste orthodoxe" effrayée par les risques d'un Grand bond en avant dans les réformes? Quoi qu'il en soit de ces deux périodes, c'est bien durant les premières années du régime qu'apparaissent le plus clairement les tentatives réformistes de Chen Yun. Loin de répondre à un a priori doctrinal, ces tentatives découlent de l'attitude flexible de Chen Yun dans la mainmise du nouveau pouvoir sur les circuits économiques, puis dans leur transformation socialiste. C'est donc à travers ces étapes successives que nous allons nous attacher à cerner la part de l'empirisme et celle du système dans le "réalisme» du personnage.

\section{Du bon usage de l'inflation : la capitulation des villes}

Lorsqu'en 1949 Chen Yun prend en main les destinées économiques de la Chine (il est alors nommé président du Comité économique et financier du gouvernement central), son souci principal est la reconstruction de la Chine, sa réunification économique. Pour réussir une telle entreprise, il se doit d'abord de juguler les comportements centrifuges des responsables locaux. Afin de faire face à la pénurie de céréales, ces responsables ont tendance à en interdire la libre circulation; les paysans, faute de pouvoir en obtenir un bon prix, s'abstiennent de vendre, et le prix des céréales augmente en ville (ajoutons que là où la réforme agraire a été accomplie, l'incitation pour les paysans à produire plus que leurs besoins afin de rémunérer des propriétaires fonciers disparaît, ainsi que le caractère automatique de la commercialisation de ce 
surplus). Le prix des céréales augmentant dans les villes, les salaires augmentent à leur tour, de même que le prix de revient des produits manufacturés : le ciseau des prix s'aggrave, les paysans peuvent acheter de moins en moins de produits industriels et leur propension à produire pour le marché diminue encore. Mais le faible prix offert aux producteurs de céréales est en soi une incitation à produire davantage de "cultures industrielles", comme le coton, dont les prix sont plus attractifs. Plus de coton signifie plus de vêtements à de meilleurs prix, et donc une amélioration du ciseau des prix - sans pour autant que soit résolu le problème des céréales.

Dans un discours du 4 avril $1951^{1}$, Chen Yun développe une approche qui n'est pas sans rappeler certains aspects de la smytchka du Boukharine de $1925^{2}$ : stimuler l'intérêt financier des paysans afin de développer la production agricole et, partant, la production industrielle. Ces incitations financières lui paraissent d'autant plus importantes qu'une grande partie des recettes budgétaires, ainsi que le financement des importations de machines, sont conditionnés par la production agricole : le développement économique "repose sur la tête des paysans" ${ }^{3}$. Bien entendu, il lui sera reproché de fonder l'alliance ouvriers-paysans sur les seconds et non sur les premiers, qui sont en théorie "l'avant-garde" ${ }^{4}$. Alors, faut-il réglementer ou libérer totalement la circulation des biens entre villes et campagne? En 1949 Chen Yun semble se prononcer pour la seconde option ${ }^{5}$. Toutefois, à bien regarder le contexte de l'année 1949, cette prise de position apparemment libérale est en fait une critique du protectionnisme (ziwei) des responsables locaux. Ce que réclame Chen Yun, ce n'est pas tant la liberté pour les individus de commercialiser à leur gré des céréales, du coton, etc., que la possibilité pour les services commerciaux d'opérer des transferts entre circonscriptions administratives : libre circulation oui, mais dans le cadre d'une réglementation unique et unificatrice; aucun souci 
"libéral" n'anime Chen Yun, seule la recherche d'un équilibre économique motive son projet.

Les mesures prises lors d'une conférence nationale réunie à la mi-février 1950 vont dans ce sens, et la "Décision concernant l'unification du travail économique et financier" du 3 mars 1950 présente l'essentiel des dispositions adoptées ${ }^{6}$. L'objet de ces dispositions peut être résumé par les trois points suivants :

a) intégration du système d'imposition fiscale (sauf les taxes agricoles annexes);

b) intégration du système budgétaire (les revenus financiers et les dépenses sont gérés par le gouvernement central);

c) intégration bancaire (la Banque populaire de Chine joue le rôle d'une chambre de compensation dans les transferts financiers entre organismes et/ou entreprises d'État)

Ces mesures instaurent également un système d'approvisionnement et de distribution centralisé afin de garantir une meilleure circulation des denrées principales. Elles sont complétées par la décision du 7 avril $1950^{7}$, qui impose aux services commerciaux d'État de déposer en banque leurs recettes quotidiennes, leur interdit toute utilisation de ces dépôts sans autorisation préalable des instances centrales, et n'autorise que des jeux d'écritures pour les règlements entre unités d'Etat. La mise en œuvre de cette politique a eu pour conséquence de faire décrô̂tre la quantité de monnaie disponible et la vitesse de circulation de la masse monétaire résiduelle dans des proportions considérables entre mars et juin 1950 : ainsi aurait été mis fin à une offensive spéculatrice de la bourgeoisie. S'il est vraisemblable que la crise du premier semestre 1950 s'est accompagnée de mouvements défensifs s'exprimant par des tentatives de spéculation, il est clair que le gouvernement chinois venait de mettre en ouvre un vaste projet dont l'objet premier était moins la lutte 
contre l'inflation, que la capitulation des villes et le contrôle de l'industrie et du commerce.

Résumons la version officielle. Le gouvernement chinois, sous l'influence de Chen Yun, se serait lancé dans une politique déflationiste drastique. Alors que la bourgeoisie avait acheté grâce au crédit bancaire, malgré de forts taux d'intérêt, les denrées disponibles dans les magasins d'État et les avait stockées, la politique commerciale, en maintenant artificiellement basses les marges bénéficiaires, l'aurait poussée à tenter de liquider, même à perte, ses stocks, qui n'auraient pu trouver preneur faute de disponibilités monétaires; d'où faillites, aggravation du chômage et baisse générale des prix à partir d'avril 1950.

En fait, cette version est trop simple et trop "pédagogique" pour être totalement satisfaisante : les stocks constitués étaient très faibles, selon Chen Yun, et, si nous acceptons l'évaluation qu'il donne de leurs moyens, les banques privées n'auraient pas été capables de financer une telle spéculation. Si le système bancaire devait jouer un rôle, ce sont les banques d'État qui auraient pu le jouer en prêtant de l'argent au commerce privé; or il leur était interdit de consentir des prêts depuis novembre 1949. En revanche, il est évident que les "larges masses" ont activement participé à ce mouvement spéculatif en constituant des stocks - si tant est qu'on puisse parler de spéculation lorsqu'il s'agit de se débarrasser, en échange de denrées indispensables, d'une monnaie dont le pouvoir d'achat s'effrite à un rythme soutenu. Qu'a-t-il pu se passer en réalité?

Au cours de l'année 1949, l'avance des armées communistes a été soutenue par l'émission continue d'une grande quantité de "monnaie populaire" (renmin bi), tant et si bien qu'entre juillet et novembre la masse monétaire - renmin bi seulement, à l'exclusion des monnaies locales en circulation en Mandchourie, en Mongolie et au Xinjiang - a doublé et que les prix ont presque triplé. En 
fait il semble bien que, sous prétexte de financer les besoins de l'armée en campagne, le pouvoir communiste ait décidé de désorganiser par l'inflation l'économie des zones où elle pénétrait, en même temps qu'il effectuait sur l'arrière des ponctions tant en monnaie (emprunts forcés) qu'en nature (transferts de céréales du Nord-Est vers le front). Puis, en novembre 1949, Chen Yun propose un plan qui, sous couvert d'une politique déflationniste qui s'impose en effet, conduira l'année suivante à la reddition économique, et donc politique, des villes. Entre autres dispositions, ce plan prévoit :

1) que les entreprises d'État et les administrations arrêtent ou retardent tous leurs paiements (c'est ce point particulier que modifie et complète la décision du 7 avril); cette disposition vise, par une réduction de la masse monétaire en circulation, à gêner la trésorerie du secteur privé;

2) qu'en 1950 sera émis, essentiellement dans les villes, un nouvel emprunt forcé (les "Bons de la victoire»), dont l'effet se fera précisément sentir à partir de la fin mars 1950 ;

3) que de novembre 1949 à mars 1950 les services commerciaux d'État arrêteront d'écouler sur le marché les denrées rares, mais se porteront acheteurs dès que les prix de ces produits feront mine de diminuer, afin de les maintenir; puis, l'échéance arrivée, d'acheteurs ils deviendront vendeurs (mars est le moment où les paysans ont vendu toute leur production commercialisable). En d'autres termes, le gouvernement chinois nourrit l'inflation et se livre à une certaine spéculation pour arriver à ses fins.

La conséquence d'une telle conjonction de dispositions - auxquelles s'est ajouté un retard de plusieurs mois dans la perception de l'impôt en céréales (gongliang) dans les zones récemment libérées ${ }^{8}$ - va de soi : tous les citadins, et en particulier les petits entrepreneurs privés qui ressentent durement la cessation de ses paiements par le 
gouvernement, sont obligés de vendre leurs réserves à vil prix (vendeurs en face d'un Etat vendeur) pour souscrire à l'emprunt forcé. Remarquons que l'indice des prix de détail affiche un baisse de $10 \%$ entre la moyenne de février et celle de mars; or celle-ci n'est pas répercutée, et la valeur d'émission des emprunts continue de croître - de 34\% durant la même période -, d'où une surcharge pour l'acheteur'.

Les résultats de cette politique sont de deux ordres :

1) Réduction de l'inflation. Cette réduction a été obtenue au prix d'une déflation drastique : production des entreprises diminuée de $30 \%$, sinon de $50 \%$, en mai par rapport à janvier 1950; près de 13000 entreprises en faillite dans les dix plus grandes villes libérées; à Shanghai, les petits bazars privés ont vu leur chiffre d'affaires diminuer de $90 \%$, les grands magasins privés d'environ $50 \%{ }^{10}$. Si donc les prix de gros ont pu baisser d'avril à juin (voir Tableau 1), ce n'est pas tant parce que les causes structurelles de l'inflation ont été supprimées, mais bien plutôt parce que, faute d'un pouvoir d'achat suffisant, les biens disponibles ne pouvaient être vendus. D'ailleurs les prix recommencent à remonter dès que le gouvernement central met en place sa nouvelle politique, et si désormais le rythme de croissance des prix est plus faible, c'est que le contrôle sur l'économie est plus grand.

2) Le réajustement du commerce et de l'industrie (juin 1950). L'emploi du mot "réajustement" (tiaozheng) montre bien l'orientation générale des dispositions prises : il ne s'agit pas d'avancer dans une révolution des structures industrielles et commerciales, mais bien plutôt de consolider les résultats acquis, de "sauver" une bourgeoisie réduite à merci et de la mettre au service de la Chine nouvelle. Chen Yun est très explicite dans ses discours du 6 et du 15 juin 1950, lors d'un plénum du CC puis d'une réunion de la CCPPC : 
Comment traiter les entreprises privées? Par la mise en place de contrats de sous-traitance (jiagong) et de contrats d'achat (dinghuo), nous pourrons progressivement organiser la production et la distribution de la production des entreprises privées. (...) Autrefois la production de la société n'était pas planifiée, aujourd'hui nous allons planifier et centraliser tout ce qui peut l'être et donc favoriser les contrats d'achat. (...) Ainsi, nous les noierons dans le socialisme ${ }^{11}$.

Lorsque nous signerons des contrats de sous-traitance ou des contrats d'achat avec des entreprises privées, le calcul du prix de revient des opérations ne devra pas se faire en fonction de la situation technique propre à chaque entreprise, mais des normes standard seront calculées en fonction des conditions moyennes des entreprises de tel secteur, dans telle région ${ }^{12}$.

Le résultat de la politique lancée en novembre 1949 est clair : le gouvernement central, suivant l'avis de Chen Yun, est parvenu dans une certaine mesure à démanteler le secteur privé, tout en ligotant les entreprises intéressantes dans un structure contractuelle destinée à garantir la stabilité des prix. Cette politique va se poursuivre, et les mouvements des "trois anti" (san fan) et des "cinq anti» ( $w u$ fan) fournissent en 1951 et 1952 les moyens politiques pour continuer la socialisation du secteur privé.

\section{Pragmatisme et contrôle économique (1951-1952)}

De la fin de l'année 1951 à la fun de l'année 1952, la répartition en pourcentage du chiffre d'affaires entre secteur d'État et secteur privé pour le commerce de gros passe du rapport $34,6 / 65,4$ au rapport $63,7 / 36,3$, et pour le commerce de détail du rapport $24,5 / 75,5$ au rapport $42,8 / 57,2^{13}$. L'évolution du secteur industriel est donnée dans le Tableau 2.

La position de Chen Yun vis-à-vis des mouvements des "trois anti" et des "cinq anti" est assez clairement exposée dans son rapport du 11 juin 1952 présenté lors d'une 
réunion de travail sur la politique de Front uni ${ }^{14}$. D'un point de vue purement politique, Chen Yun approuve sans réserve l'aide que peuvent lui apporter ces mouvements dans son travail de restructuration de l'économie et regrette même qu'ils n'aient pu être lancés dès 1950 . D'un point de vue purement économique, en revanche, il est beaucoup plus réticent, car il est obligé de constater que ces mouvements politiques perturbent grandement la bonne marche de l'économie, à qui l'on doit pratiquer la «respiration artificielle». C'est pourquoi il se montre partisan de méthodes plus douces et, à son sens, tout aussi efficaces, sinon plus; il se prononce par conséquent pour des modes économiques de contrôle, plutôt que des modes politiques encourageant les excès de zèle.

Ce pragmatisme et cette volonté de contrôle économique se retrouvent dans toutes les dispositions que Chen Yun inspire jusqu'à la fin de l'année 1952. Au cours des premières années du régime, la planification n'existait encore que de façon embryonnaire : diversité des systèmes monétaires (la Banque de Mandchourie et la Banque de Mongolie ne seront transformées en succursales de la Banque populaire de Chine qu'à compter du ler avril 1951 , et les petites banques privées ne seront «socialisées" qu'en décembre $1952^{15}$ ); manque de personnel qualifié; manque d'expérience pratique; mais aussi importance du secteur privé - industriel, commercial, aussi bien qu'agricole -, impossible à intégrer dans une planification globale. Dans cette conjoncture, l'introduction du contrôle gouvernemental dans la distribution des matières premières et dans l'attribution des crédits, ainsi que l'établissement de liens contractuels entre le secteur privé et l'État, sont autant de moyens pour "socialiser" ce secteur et pour lui imposer un mode de supervision de plus en plus proche de celui qui est mis en place dans le secteur d'État.

Dans le même temps, par une ordonnance en date du 29 avril 1951 , le gouvernement chinois tente de rappro- 
cher le mode de gestion micro-économique du secteur d'État de celui du secteur privé. II décide en effet d'attribuer aux entreprises industrielles d'État la personnalité comptable (jingji hesuan); de même, par un décret en date du 1er juin 1951, le Comité économique et financier décide d'apurer les comptes de l'ensemble des entreprises du secteur d'État avant de leur attribuer à toutes la personnalité comptable ${ }^{16}$. L'introduction de cette dernière s'accompagne du droit de solliciter directement des emprunts auprès des banques d'État, qui assumeront un rôle de contrôleur; de l'obligation pour les dirigeants d'entreprises d'assumer la responsabilité des pertes de leur unité; du droit, une fois le plan accompli, d'acquérir des matières premières et de commercialiser directement la production hors quota; et enfin, de l'instauration d'un système de primes prélevées sur le profit hors quota, dans la limite de $30 \%$.

En exigeant des dirigeants des entreprises du secteur d'État qu'ils tiennent des comptes et présentent leur bilan, et en introduisant un système de stimulants matériels ainsi qu'un embryon de système de responsabilité, la personnalité comptable est supposée offrir à la fois une incitation à produire et assurer un contrôle de cette production, et partant, son adéquation au Plan d'État.

La mise en place de ce système de gestion emprunté aux Soviétiques, le khozrastchêt, ne s'est pas faite sans problèmes, en particulier dans le secteur commercial où elle a été retardée afin de faire face aux exigences de la guerre de Corée. Dans le cas du commerce, la liaison entre le renforcement du contrôle économique sur les entreprises d'État et la limitation du contrôle politique local est claire : le découpage territorial et le regroupement des entreprises commerciales ne doivent plus épouser les frontières des grandes régions administratives ( $d a$ xingzheng $q u$ ), mais celles des régions économiques (jingji $q u)^{17}$. Cette décision de septembre 1952 précède de peu deux décrets organiques de novembre 1952 allant dans le 
sens d'une plus grande centralisation économique ${ }^{18}$. Le premier stipule la transformation des gouvernements régionaux en commissions administratives. Cette mesure, cependant, ne revient pas sur la déconcentration administrative de mars 1951, conçue pour lutter contre l'«esprit d'indépendance" (dulixing) et l'«individualisme» (benwei zhuyi) des responsables locaux, et qui instaurait trois échelons budgétaires : le Centre, la grande région administrative, et la province ${ }^{19}$; dès cette époque, Chen Yun prévoyait de donner la personalité budgétaire au xian à dater de $1952^{20}$. Le second décret, quant à lui, crée une Commission d'État au plan, province de Gao Gang dont l'élévation semble être allée de pair avec un certain recul de Chen Yun.

\section{Gao Gang contre Chen Yun}

La présidence de cette nouvelle Commission est donc confiée, le 16 novembre, à Gao Gang. Cette nomination, même si elle s'accompagne d'une limitation du contrôle politique direct de Gao Gang sur la Mandchourie, ne semble pas alors pouvoir être interprétée comme un signe de méfiance à son égard : n'est-ce pas lui qui, dans un article du Renmin ribao du ler octobre 1952, décrit la politique à mettre en ouvre pour l'industrialisation de la Chine? N'est-ce pas encore lui qui est qualifié de "proche compagnon d'armes" de Mao Zedong au début de 1953? De même que Gao Gang, qui représente la Chine de l'industrie lourde, Rao Shushi, qui représente la Chine de l'industrie légère, est appelé de Shanghai au Centre. En fait, celui qui semble le grand perdant dans l'affaire est bien Chen Yun, qui perd sa prééminence économique sans pouvoir prétendre, en contrepartie, jouer les éminences grises au sein d'une Commission destinée à faire office de véritable gouvernement économique parallèle. La liste des membres de cette Commission (voir Tableau 3) n'offre pas l'image d'une très grande homogénéité, si l'on ex- 
cepte peut-être une commune opposition potentielle à Mao Zedong : de fait, elle fait penser à une liste de condamnés préparée à l'avance, le seul rescapé étant Zhang Xi... décédé de mort naturelle en 1959. Mais ce manque d'homogénéité dans les personnalités est sans doute la richesse d'une telle assemblée. Notons aussi que près de la moitié des membres de la Commission ont, à un titre ou à un autre, exercé des responsabilités en Mandchourie après 1946; ils sont donc parfaitement conscients de l'importance de cette région tant du point de vue économique (en tant que moteur de la reconstruction et base du développement) que politique (réforme agraire...). En outre, près de la moitié également des membres sont des techniciens de l'économie.

L'absence, du moins apparente, de critiques quant à la purge de Gao Gang (rendue publique en mars 1955) laisse penser qu'il existait effectivement une certaine unanimité contre le personnage. Le fait que personne n'ait été accusé par les Gardes rouges d'avoir soutenu Gao Gang renforce cette hypothèse ${ }^{21}$. La lenteur du processus de la purge (deux ans) est-elle liée à la place particulière de la Mandchourie?

Sa place dans les relations sino-soviétiques d'abord. Peut-on soutenir que la nomination de Gao Gang résultait d'un marchandage entre les Chinois et les Russes - ces derniers n'acceptant d' "aider" la Chine qu'à la condition de pouvoir contrôler l'usage qui était fait de cette aide? La nouvelle Commission n'a-t-elle pas été mise en place juste après le retour de Moscou d'une délégation chinoise menée par Zhou Enlai et Chen Yun, partie solliciter une aide économique accrue auprès du "Grand frère", et n'avait-elle pas précisément pour mission de gérer cette aide? Rappelons qu'il existait déjà une cellule de planification au sein du Comité dirigé par Chen Yun, mais que cette cellule a été supprimée lors de la création de la Commission du plan. C'est aussi à cette occasion qu'est créé le Ministère numéro un des machines-outils (sous la 
direction de Huang Jing) pour gérer les machines-outils de base, en particulier celles importées d'URSS. N'est-ce pas à Li Fuchun (ministre de l'industrie lourde jusqu'en septembre) et à Wang Heshou (compagnon de Li Fuchun au Xibei, responsable de l'industrie lourde en Mandchourie, puis ministre de l'industrie lourde à la suite de $\mathrm{Li}$ Fuchun) qu'est laissé le soin de négocier dans le détail les modalités de cette aide? Li Fuchun est en outre nommé vice-président de la Commission dès sa création, avant d'en devenir, selon certaines sources, le président de facto une fois Gao Gang purgé, puis de jure en septembre 1954 .

La place de la Mandchourie dans le processus de reconstruction économique de la Chine explique également la prééminence des cadres qui en proviennent. En décembre 1949 le Nord-Est n'est-il pas érigé en base pour le développement de la sidérurgie en Chine, bénéficiant à ce titre d'investissements prioritaires ${ }^{22}$ ? Il y a sans nul doute une forte interaction entre la politique économique menée en Mandchourie dès 1946 et celle prônée pour toute la Chine à partir de 1949. En effet, les artisans de la remise en ordre de l'économie du Nord-Est sont Chen Yun et $\mathrm{Li}$ Fuchun, et il était naturel que l'on recoure aux recettes appliquées avec succès en Mandchourie pour tenter de guérir les mêmes maux dans le reste de la Chine $^{23}$. Lorsque, en octobre 1952, donc avant que la nouvelle Commission ne soit effectivement en activité, le Comité économique et financier se réunit pour décider de la politique de construction de base pour 1953, la Mandchourie est à la fois pôle et modèle de développement pour l'économie du pays ${ }^{24}$. De même, les discussions sur les modes de gestion et de contrôle de l'économie tournent toutes autour d'expériences imitées des Soviétiques et menées en Mandchourie : cela tient au rôle économique de la région, indépendamment de la personnalité de son dirigeant. Même si Gao Gang était la "carte" jouée par les Soviétiques pour asseoir leur influence en 
Chine; même s'il avait (et c'est loin d'être évident) ses propres conceptions économiques; même s'il comptait utiliser l'URSS pour affirmer son influence (témoins son accord commercial "Soviéto-mandchou" et ses déclarations en faveur d'une réunion des deux Mongolies sous l'égide de l'URSS...) - il est malgré tout très vraisemblable que si les dirigeants chinois ont décidé de le déplacer au Centre, ce n'était pas simplement pour mieux juguler ses ambitions, c'était surtout pour se ménager les bonnes grâces de l'URSS et de Staline ${ }^{25}$.

$\mathrm{Si}$ ces différents aspects justifient la nomination de Gao Gang, en revanche ils n'expliquent pas la relative mise à l'écart de Chen Yun, lequel n'est même pas nommé vice-président de la nouvelle Commission ${ }^{26}$, sauf à supposer que celle-ci n'aurait eu qu'un rôle purement politique et que Chen Yun et Mao Zedong, agissant de concert, auraient tous deux "planifié" la chute de Gao Gang. Cette hypothèse ne semble pas entièrement dénuée de fondement si l'on considère le déroulement de la Conférence nationale sur le travail économique et financier, réunie du 13 juin au 12 août 1953, c'est-à-dire dans un contexte politique très particulier : celui de la mort de Staline, suivie quelques jours plus tard (le 26 mars) de la signature d'un accord économique sino-soviétique, puis, en juillet, de la conclusion de la guerre de Corée, qu'elle a précisément facilitée.

L'objet de cette conférence était la définition de la "ligne générale pour la transition vers le socialisme". La direction économique semble avoir été soumise à des critiques venant de responsables tant locaux que centraux, Mao Zedong en tête. Deux rapports importants y sont présentés, l'un par un responsable économique (Jia Tuofu?) sur la planification de la construction économique, l'autre par un responsable du Département du Front uni (Li Weihan?). Zhou Enlai en établit le bilan. Chen Yun et Mao Zedong $y$ interviennent également ${ }^{27}$. 
Or à cette occasion se serait manifestée avec éclat l'opposition entre Mao Zedong et Gao Gang ${ }^{28}$ : Staline mort, ce dernier a perdu son soutien le plus précieux, cependant que Malenkov et Khrouchtchev, chacun pour son propre compte, courtisent Mao Zedong; celui-ci, dans un premier temps, accorde ses faveurs à Khrouchtchev, avant de les vendre, en septembre 1954, à Malenkov, qui non seulement les paye en nature - renonciation aux vues soviétiques sur la Mandchourie -, mais aussi en espèces 520 millions de roubles d'aide et de crédits $^{29}$.

Mais revenons au lendemain de la mort de Staline et de la signature de l'accord sino-soviétique du 26 mars. Le 27 mars, Jia Tuofu, vice-président du Comité économique et financier et de la Commission d'État au plan, réunit sous sa présidence un certain nombre de responsables économiques de ces deux administrations afin d'organiser des équipes de contrôle dans plusieurs ministères (industrie lourde, machines-outils numéros 1 et 2 , combustibles et chemins de fer), qui les aideront à planifier la construction économique; en d'autres termes, à répartir l'aide soviétique. Les options définies sous la direction de Jia Tuofu peuvent se définir par la formule suivante : prendre l'industrie lourde pour base et la Mandchourie comme modèle ${ }^{30}$. C'est vraisemblablement ce programme, à l'élaboration duquel Chen Yun ne semble pas avoir pris part, qui est présenté, sans doute par Jia Tuofu, lors de la conférence nationale de l'été 1953 , et qui rencontre l'opposition de Mao Zedong et de Chen Yun, quoique sans doute pour des raisons différentes. En effet, comme nous le verrons, seule cette alliance temporaire peut expliquer l'indulgence dont Mao Zedong fait preuve cet été-là à l'égard de Chen Yun, et la confiance qu'il lui manifestera six mois plus tard, en mars 1954. (Deng Xiaoping indique que ce serait à l'initiative de Chen Yun que lui-même et Chen auraient incité Mao à s'opposer à Gao Gang ${ }^{31}$ ).

Le thème de l'exposé de Li Weihan ${ }^{32}$ était : comment "utiliser, confiner, transformer" (liyong, xianzhi, gaizao) 
la bourgeoisie; et les cibles du responsable du Département du Front uni étaient Bo Yibo (vice-président du Comité économique et financier et ministre des finances) et Chen Yun. Mao Zedong, dans son discours de clôture, limite quant à lui ses critiques au seul Bo Yibo ${ }^{33}$. Comment est née la crise d' "aventurisme" de 1953, qui permet au secteur privé de relever la tête?

\section{Regain et défense du secteur privé (1953-1954)}

En 1952, à la suite des mouvements des "trois anti" et des "cinq anti", le marché avait connu une crise, les produits n'arrivant plus à s'écouler. Pour revivifier le marché, le Comité économique et financier avait décidé de réformer le système fiscal dans le sens d'une répartition plus égalitaire des charges entre secteur privé et secteur d'Etat ${ }^{34}$. Et, le 31 décembre 1952, Chen Yun avait fait paraître sous sa propre signature, dans le Renmin ribao, les dispositions nouvelles. Là est précisément le point central des critiques : Chen Yun et Bo Yibo traitent sur un pied d'égalité le secteur privé et le secteur d'Etat (gong si yilü pingdeng). $\mathrm{Si}$, en 1953, Mao Zedong impute cette erreur au seul Bo Yibo, en revanche les dazibao de la Révolution culturelle accuseront Chen Yun du même péché ${ }^{35}$. En revanche les hagiographes de Chen Yun nient l'existence d'un tel acte de lèse-idéologie de sa part - les $\boldsymbol{E}$ uvres choisies répertorient seulement un texte de septembre 1953, donc postérieur, où Chen évoque le problème, mais pour approuver la condamnation d'une telle hérésie ${ }^{36}$. Cela dit, les choses seraient peut-être passées inaperçues si n'étaient intervenus d'autres facteurs ${ }^{37}$.

Une erreur de gestion budgétaire, d'abord. Fin 1952, les services financiers constatent que les sommes inscrites au budget n'ont pas été employées dans leur totalité : aussi transfèrent-ils ce solde en recettes sur le budget de 1953, mais en oubliant de transférer les dépenses 
afférentes; en d'autres termes, contrairement à la règle qui veut qu'à chaque yuan de recette corresponde un yuan, et un seul, de dépense, certaines sommes ont deux emplois (yi nü er jia) : l'un prévu par le budget de 1952, l'autre décidé par le budget de 1953. Par suite, le volume de l'investissement en construction de base peut augmenter de $50 \%$, et dès juin 1953 , le déficit budgétaire atteint plus de deux milliards de yuan. Les choses en seraient peut-être restées là si, devant cet afflux de liquidités, les banques n'avaient accepté d'accorder au secteur privé des crédits à des taux d'intérêts bénéficiant d'une baisse supérieure à celle des taux appliqués au secteur d'État, et n'avaient de ce fait encouragé le développement du secteur privé au détriment du secteur d'État.

Et ensuite, l'introduction de la personnalité comptable dans les entreprises commerciales. La mise en place de ce système de gestion exigeait des entreprises commerciales d'État qu'elles fassent l'inventaire de leurs stocks afin de déterminer leur niveau optimum, et donc d'évaluer leurs besoins réels en fonds de roulement (dans l'idée du projet, "évaluer" est synonyme de "réduire»). Dans la conjoncture de la fin de 1952 et du début de 1953 stocks importants dus à la mévente -, cela conduisait les entreprises à sous-évaluer la demande sur le marché, à estimer leur stock optimum à un niveau très bas, et à refuser d'acquérir une partie, ou même la totalité, des produits fabriqués par les entreprises industrielles : ainsi purent-elles réévaluer en baisse leurs besoins en fonds de roulement et rembourser aux banques une partie de leurs avances en trésorerie - d'où nouvel afflux de liquidités. Quant au secteur commercial privé, non seulement il pouvait bénéficier facilement d'avances de trésorerie bon marché, mais encore il se trouvait en face d'unités de production industrielle désireuses, faute de pouvoir vendre aux entreprises commerciales d'État, de trouver des acheteurs de remplacement. De façon très logique, le secteur privé était en train de renaître de ses cendres au 
détriment du secteur d'État (gong tui si jin); d'où la colère des tenants de la ligne dure à l'égard de la bourgeoisie.

D'un point de vue politique, la conférence de l'été 1953 a pour conséquences les nominations, le 18 septembre de cette année, de Deng Xiaoping au poste de ministre des finances à la place de Bo Yibo, et de $\mathrm{Li}$ Weihan à celui de vice-président du Comité économique et financier présidé par Chen Yun. Ce dernier indiquera en $1962^{38}$ que l'une de ses principales fonctions au début du régime avait été de s'occuper de la socialisation du secteur privé. L'arrivée de $\mathrm{Li}$ Weihan (le directeur du Département du Front uni) au Comité économique et financier était en fait un désaveu du travail effectué par Chen Yun, et signifiait un transfert des responsabilités du second au premier en matière de socialisation. C'est au double titre de directeur du Département du Front uni et de vice-président du Comité économique et financier chargé de la socialisation que $\mathrm{Li}$ Weihan présidera, en novembre 1953, à la naissance de la Fédération de l'industrie et du commerce [privés].

Chen Yun commence sans doute à payer le prix du soutien de Mao Zedong dans l'affaire de la réforme fiscale lors de la réunion du Bureau politique du 24 décembre 1953. Mao exige alors l'élimination de Gao Gang et décide de prendre des "vacances» jusqu'à ce que satisfaction lui soit accordée. Le quatrième plénum (élargi) du 5e CC, qui se tient du 6 au 10 février 1954, lui offre effectivement la tête de Gao Gang (lequel n'était plus apparu en public depuis le 26 décembre de l'année précédente), ansi que celles de Rao Shushi et de sept autres comparses; et Mao réapparaît publiquement le 24 mars ${ }^{39}$. C'est alors une - brève - période de gloire qui commence pour un Chen Yun qualifié de «proche compagnon d'armes» de Mao Zedong - comme un an plus tôt Gao Gang ${ }^{40}$ ! En mars 1954, donc au retour de Mao, il est chargé de l'élaboration du premier plan quinquennal. Il 
s'agit de la cinquième rédaction, les trois premières ayant été composées avant 1953 par le Comité économique et financier (dont la cellule de planification était alors dirigée par Song Shaowen), et la quatrième par la Commission au plan présidée par Gao Gang ${ }^{41}$. Le 30 juin, Chen Yun transmet un rapport sur son projet de premier plan quinquennal au Comité central. Mais, comme on sait, il n'y a qu'un pas du Capitole à la Roche tarpéienne, et Chen Yun l'a franchi dès le 25 mai 1954.

Cet épisode, qui n'est pas répertorié dans l'histoire officielle, nous est connu grâce à la Révolution culturel$\mathrm{le}^{42}$. Le $25 \mathrm{mai}$, lors de la 2e conférence nationale sur la propagande et de la $6 e$ conférence nationale sur la sécurité publique, Chen Yun présente un rapport sur «Le problème du commerce» (Guanyu shangye wenti), dans lequel il aurait fait les déclarations suivantes :

La croissance du pouvoir d'achat dans la société est supérieure à celle de la production de biens de consommation. Cette tendance est loin d'être provisoire. L'URSS connait toujours cette situation où l'offre est inférieure à la demande. (...) Aujourd'hui, d'où vient principalement ce déséquilibre? (...) La population paysanne est la plus nombreuse, l'économie agricole est la plus importante : aussi la responsabilité de ce déséquilibre revient-elle en grande partie aux paysans. (...) Les prix des produits agricoles ne peuvent qu'augmenter et les prix des produits industriels ne peuvent que diminuer. (...) A l'heure actuelle nous ne pouvons avancer [dans la socialisation]; nous devons marquer un arrêt, [sans quoi] toutes les boutiques vont fermer. (...) Nous devons laisser aux commerçants de quoi manger, (...) les rassurer (anding renxin). (...) Les entreprises locales, d'État ou mixtes, sont rassasiées; en revanche, les entreprises privées sont affamées. (...) Le secteur capitaliste et le secteur socialiste, compte tenu de leurs différences, doivent être traités sur un pied d'égalité. (...) Le secteur commercial et industriel capitaliste doit faire des produits rationnels, sinon ce serait injuste. 
Cette condamnation de la poursuite - pour l'heure - de la socialisation, ainsi que l'exigence proclamée d'un meilleur traitement du secteur privé, paraissent plus que vraisemblables de la part de Chen Yun, car, si son rapport du 25 mai 1954 n'a pas été rendu public, en revanche nous connaissons trois autres documents de 1954 dans lesquels nous retrouvons, sur un mode mineur, les mêmes préoccupations ${ }^{43}$. Mais la meilleure approche et la meilleure présentation (indirecte) de sa position se trouvent encore dans un texte de Ma Yinchu rédigé entre janvier et avril 1955 : La transformation socialiste de l'industrie chinoise $^{44}$. En mai 1955, Ma transmet le manuscrit de son texte à Chen Yun pour approbation, et ce dernier en profite pour apporter une modification importante au passage juste cité de son discours du 25 mai 1954, qui avait alors particulièrement choqué Mao Zedong et les tenants d'une ligne dure à l'égard du secteur privé.

Dans la version originale, comme on l'a vu, Chen Yun déclarait que le secteur capitaliste et le secteur socialiste, *compte tenu de leurs différences, doivent être traités sur un pied d'égalité" (ying zai you suo butong $z h i$ xia, yi shi tong ren). Après relecture du manuscrit de Ma Yinchu, et dans une lettre à l'auteur, Chen suggère, en 1955, de corriger cette phrase comme suit : le secteur capitaliste et le secteur socialiste, "malgré leurs différences, peuvent être soumis au même régime dans certains cas" (jiyao you suo butong, you yao zai yixie fangmian yi shi tong ren $)^{45}$.

En d'autres termes, la formulation initiale signifiait qu'il fallait conserver aux deux secteurs leurs caractéristiques et, partant, leur utilité propre sans pénaliser l'un par rapport à l'autre; Chen demandait donc aux dirigeants chinois d'accepter l'existence, sinon la survivance, d'un secteur privé parallèlement au secteur d'État et au secteur mixte : il persistait dans son hérésie égalitariste de décembre 1952. En revanche, la formulation corrigée est ambiguë, car elle peut laisser penser que Chen Yun a 
changé d'opinion alors que seules les circonstances ont changé : le 2 septembre 1954, lors d'une réunion du gouvernement, $\mathrm{Li}$ Weihan avait présenté un projet de décret pour la socialisation du secteur industriel privé par la mixtion des secteurs (gong si heying), dont la justification économique aurait été la nécessité de renforcer la planification centralisée. Ce décret est promulgué le jour même ${ }^{46}$, et il ferait suite à un texte semblable concernant le commerce ${ }^{47}$. Dans ce contexte nouveau, la phrase "corrigée" de Chen Yun peut être interprétée (et c'est le propos de $\mathrm{Ma}$ Yinchu) comme une mise en garde contre une socialisation trop rapide : bien qu'il existe des différences d'objectif entre les entrepreneurs nommés par l'Etat et les entrepreneurs privés - c'est l'opposition entre intérêt public et privé -, il peut néanmoins arriver que dans certains cas les nécessités économiques (ici, le renforcement de la planification) exigent effectivement de procéder à une socialisation de tel ou tel secteur par le biais de la constitution d'entreprises mixtes - ce qui revient à imposer un même régime à l'ensemble du secteur.

Or, malgré cette concession, il semble bien que Chen Yun n'ait pas été convaincu que le renforcement de la planification passait inéluctablement par la socialisation immédiate du secteur privé. Essayons de reconstituer son argumentation au travers des sources déjà indiquées.

Pour une régulation plus flexible

La constatation de départ de Chen Yun est ce déséquilibre entre la croissance du pouvoir d'achat et celle de la production de biens de consommation, déséquilibre qu'il impute aux paysans qui se refusent à commercialiser leurs surplus en céréales, en oléagineux, etc., qu'ils préfêrent consommer plutôt que vendre. Claude Aubert, dans son "modèle hypothétique" de consommation des grains alimentaires, montre bien la double tendance de la 
période 1952-1957 : une augmentation progressive de la consommation rurale et une diminution de la consommation urbaine, sans que l'on puisse attribuer cette dernière évolution à un changement des habitudes alimentaires ${ }^{48}$. Cette situation justifie donc, pour Chen Yun, l'introduction d'un système d'achat et de vente unifié (tong gou tong xiao) en novembre $1953^{49}$. Or, changer de système d'approvisionnement revient seulement à changer de problème, car désormais, ce que les paysans gardent par devers eux ce sont des liquidités monétaires, qu'il convient de résorber. Plusieurs solutions sont envisageables : aggravation du ciseau des prix, emprunt forcé, mais aussi augmentation de la production industrielle. C'est cette dernière que retient Chen Yun, sans pour autant rejeter les autres. Du coup de nouveaux problèmes se posent :

a) La production de biens de consommation. Dans ce domaine, le secteur privé a un rôle à jouer comme adjuvant du secteur étatisé, un rôle qui peut être orienté, voire planifié, grâce à la politique contractuelle (jiagong dinghuo). Mais le principal problème ici est l'incapacité des usines et ateliers chinois, tant privés que publics, à produire pour l'heure les machines-outils qui permettraient de fabriquer de nouveaux produits. C'est pourquoi l'attention du gouvernement doit se porter en priorité sur la politique d'investissement, afin que soit évitée la multiplication de petites unités de production inadaptées qui entreraient en concurrence avec celles existant déjà (privées ou non), sans pour autant aider à accroître l'offre en biens de consommation. Dans ce contexte, la socialisation du secteur privé ne saurait constituer une solution, et ce d'autant moins que les entrepreneurs privés volontaires pour entrer dans des entreprises mixtes (gong si heying) sont généralement ceux dont les entreprises battent de l'aile.

b) La commercialisation des biens de consommation. La suppression du secteur commercial capitaliste, lequel est 
indispensable dans les relations villes-campagnes, priverait la Chine d'un réseau de distribution unique qu'elle a les moyens de contrôler par une politique contractuelle (dai xiao et jing xiao). En outre, la disparition d'un tel réseau favoriserait la persistance des tendances centrifuges de la part des autorités locales, qui verraient là un prétexte pour investir dans les secteurs où Chen Yun estime précisément que ce serait du gaspillage (par effet de redondance). On en revient ainsi au problème précédent.

Si la socialisation du secteur privé n'est pas une solution, il est absurde d'exercer sur lui des pressions comme celles qu'il subit en ce premier semestre 1954. Au contraire, il faut lui garantir une juste rémunération pour s'assurer de son concours. Quant au renforcement de la planification du développement industriel, il doit passer par une meilleure utilisation des unités de production existantes dans les bases industrielles anciennes, par une meilleure gestion et par une orientation plus adéquate de l'investissement productif.

Cette prise de position vaut à Chen Yun la perte de sa prééminence économique lors de la création du Conseil des affaires de l'État (CAE) en septembre 1954. Certes, dans la nouvelle structure gouvernementale, Chen Yun reste vice-premier ministre - le premier des dix ayant ce titre, et en tant que tel appelé à remplacer Zhou Enlai lors de ses voyages à l'étranger. Mais les fonctions de superviseur de l'économie qu'il pouvait exercer au travers du Comité économique et financier sont désormais réparties entre les personnes suivantes :

- Li Xiannian, vice-premier ministre, ministre des finances et directeur du 5e bureau du CAE (finances, commerce intérieur et extérieur);

- Li Fuchun, vice-premier ministre et président de la Commission d'État au plan, dont l'un des vice-présidents, Xue Muqiao, est directeur du Bureau national des statistiques; 
- Bo Yibo, président de la Commission d'État à la construction de base et directeur du 3e bureau du CAE (investissement);

- Li Weihan, chef du Département du Front uni, directeur du 8e bureau du CAE (bourgeoisie nationale et minorités). Dans ces deux fonctions, Li a comme adjoint l'économiste marxiste Xu Dixin, également responsable du Service central du commerce et de l'industrie. (Chen Shutong et Sha Qianli, respectivement président et secrétaire de la Fédération chinoise pour le commerce et l'industrie [privés], contrôlée par le Front uni, sont, l'un, vice-ministre des céréales, et l'autre, ministre de l'industrie locale [privée]).

Il est intéressant de noter que ceux qui remplacent Chen Yun dans ses principales fonctions économiques appartiennent au groupe que Roderick MacFarquhar appelle les «planificateurs" (planners). Or, bien que ceux-ci se soient souvent montrés plus sensibles aux arguments économiques de Chen Yun qu'à ceux de Mao Zedong, leur carrière politique, contrairement à celle de Chen lui-même, n'en a pas souffert pour autant, du moins jusqu'à la Révolution culturelle. Il est vraisemblable que cette différence s'explique par la personnalité des individus : le contraste entre l'attitude des uns (soumise et résignée) et celle de l'autre (intransigeante) lors de la préparation et du lancement du Grand Bond en avant ne suggère-t-il pas une relation de cause à effet entre "caractère» et avenir politique? En cette année 1954, Chen Yun se trouve désormais confronté, sinon à l'opposition directe des planners, du moins à leur inertie potentielle.

Une dernière tentative en faveur du marché : Chen Yun dans la crise de 1956

Bien que l'action de Chen Yun en faveur d'une politique moins brutale à l'égard du secteur privé soit bloquée par le réseau animé par $\mathrm{Li}$ Weihan, les faits vont lui donner 
malgré tout la possibilité de réaffirmer la nécessité d'une approche plus réaliste. En 1955, la poursuite accélérée de la socialisation, en particulier celle du petit commerce de détail privé, suscite rapidement des inquiétudes en raison de la rapide diminution du nombre des petits commerçants dans les campagnes. Le nombre de cadres dans les villages et les bourgs de moins de 20000 habitants s'élève à huit cent mille; or, 3600000 personnes sont nécessaires pour assurer le bon fonctionnement du réseau commercial dans les villages et les bourgs, soit un chiffre correspondant grosso modo au nombre des cadres augmenté de celui des petits commerçants ruraux. La disparition de ces derniers, même si elle se fait toujours par intégration dans les services commerciaux d'État, ne peut être que préjudiciable aux bonnes relations entre villes et campagnes ${ }^{50}$. La situation semble avoir évolué vers un tel degré de confusion que la socialisation de l'industrie et du commerce va être un des principaux thèmes analysés lors de la $3 \mathrm{e}$ session plénière de l'Assemblée nationale populaire en juin 1956.

Il était certes impossible de revenir sur une politique décrite comme un succès total, les maladresses dans la mise en œuvre étant imputées aux seules actions malivoles des capitalistes au sein des entreprises mixtes ${ }^{51}$. Bien qu'identifiant des erreurs plus objectives, Chen Yun se doit de mouler ses discours des 18 et 30 juin $1956^{52}$ dans cette rhétorique s'il veut avancer ses propositions en vue d'une libération limitée du marché libre, en particulier dans les zones rurales. Il sait d'ailleurs convaincre, et des expériences en ce sens sont lancées en août 1956 dans neuf provinces (Hubei, Guangdong, Shanxi, Shandong, Jiangxi, Sichuan, Hebei, Fujian et Jiangsu). Ce succès lui permet de retrouver une fonction ministérielle précise (le commerce) en novembre 1956.

Chen Yun a entre temps exposé dans le détail ses conceptions dans un important discours prononcé le 20 septembre devant le VIIle Congrès du Parti ${ }^{53}$. La lecture 
de ce discours montre qu'il y prend les précautions indispensables pour justifier son action, puisqu'il spécifie que le résultat

ne sera aucunement la réapparition en Chine d'un marché capitaliste, mais la naissance d'une sorte de marché socialiste répondant à la situation du pays et aux besoins du peuple. La situation de notre économie socialiste sera la suivante. Concernant la distribution et la production industrielle : le secteur d'État et le secteur collectif auront la prééminence dans le commerce et l'industrie, mais en concurrence avec un secteur individuel annexe. Ainsi, le secteur individuel soutiendra le secteur d'État et le secteur collectif. Concernant la planification de la production : la majeure partie de la production agricole et industrielle chinoise sera soumise à la planification, mais dans le même temps un certain nombre de produits seront fabriqués librement en suivant les changements du marché, et dans les limites spécifiées par le plan. (...) Ce type de marché en économie socialiste n'est nullement le marché libre capitaliste, c'est un marché unifié socialiste. Dans ce marché unifié socialiste, le marché d'État a la prééminence, mais en concurrence avec un marché libre sous la tutelle de l'État. Ce marché libre sous la tutelle de l'État est un complément au marché d'État, c'est pourquoi il est un élément constitutif du marché socialiste unifié.

Plus concrètement, l'argumentation de Chen Yun enchaîne cinq propositions principales :

1) Les dispositions antérieures avaient pour but de contrôler le commerce et l'industrie privés afin d'éviter des mouvements spéculatifs; a ujourd'hui qu'il n'existe plus de secteur privé, ces dispositions sont non seulement inutiles, mais encore fâcheuses. (Notons que, ce disant, Chen Yun ne remet nullement en cause la socialisation, même s'il a pu en trouver le pas trop rapide).

2) Les services commerciaux distingueront deux types de produits. En ce qui concerne les produits importants comme les céréales, les cotonnades, le charbon, etc., c'est le système d'achat et de vente unifiés qui doit être 
appliqué. En ce qui concerne les autres produits, les services d'État ont un droit de préemption, et les entreprises peuvent commercialiser librement ceux qu'ils n'ont pas jugé nécessaire d'acquérir. L'approvisionnement des entreprises sera assuré à la fois par le marché et par la politique de répartition de l'État. Par ailleurs, pour assurer une meilleure circulation des produits entre régions et provinces, les services de gros n'auront pas le droit d'imposer leurs marchandises aux unités qui leur sont subordonnées territorialement, lesquelles pourront s'approvisionner librement.

3) Afin de mieux répondre aux besoins du marché, il convient que les nombreuses petites unités de production industrielle, artisanale, agricole (produits annexes) et commerciale soient éparpillées, et donc que soit mis un frein au regroupement systématique en grosses unités, ce qui conduit à une diminution de la variété et de la qualité des produits.

4) La politique des prix ne doit pas se limiter à une unification ou à un gel des prix. Des variations temporaires, même à la hausse, n'ont rien d'inquiétant. Les prix doivent être déterminés en fonction de la qualité et des conditions objectives. En d'autre termes, (a) les produits de qualité supérieure, d'un prix de revient souvent plus élevé, doivent être vendus plus cher afin de prévenir une baisse de qualité; (b) les produits nouveaux doivent aussi pouvoir être commercialisés, au début, à un prix de vente élevé, afin que la production en soit encouragée; le développement de la production conduira à une baisse des prix de revient, donc, à terme, des prix de vente; (c) les produits locaux commercialisés librement verront leurs prix augmenter; cette hausse, conduisant à une augmentation de la production et donc à la satisfaction de la demande, n'est pas à craindre : c'est au contraire une politique irrationnelle des prix qui provoquerait des tensions sur le marché. 
5) La planification de la production des produits de consommation courante doit être considérablement assouplie afin que les unités de production, qui sont pieds et poings liés par des normes impératives, ne soient pas conduites à se soucier seulement de leurs résultats financiers. Ces normes doivent avoir un simple caractère de référence, utile tant aux services de planification qu'aux entreprises elles-mêmes, lesquelles pourront adapter leur production aux besoins du marché.

Nous laissons au lecteur le soin d'apprécier les convergences de ce programme avec la politique suivie depuis 1978... En 1956, malgré une amélioration de la circulation de certaines denrées, des dérapages vont très vite se faire sentir. C'est ainsi que Ma Yinchu notera par la suite ${ }^{54}$ :

In est de fait que, depuis l'élargissement du marché libre, certains inconvénients s'étaient manifestés. Ainsi, nombre de produits agricoles annexes dont la demande était supérieure à l'offre avaient-ils vu leurs prix augmenter brutalement. Les prix sur le marché s'établissaient à un cours supérieur aux prix réglementés, et nombre de paysans refusaient de vendre ces produits aux services étatiques, allant même jusqu'à dénoncer leurs contrats de vente, à rembourser leurs avances, etc. Le comportement habituel des paysans était ressorti. Les causes de cette situation résultaient principalement du manque de direction de la part des administrations concernées sur la part élargie du marché libre, d'un développement plus rapide de la pratique sur le travail de propagande, et d'une compréhension insuffisante, de la part de nombre de cadres et de celle des masses, du domaine et de la signification de l'élargissement du marché libre.

Aussi, dès octobre 1956, le gouvernement chinois tente-t-il de redéfinir le cadre du marché libre ${ }^{55}$ en convoquant, le 25 de ce même mois, une conférence sous la tutelle de Li Xiannian. En janvier 1957, il est mis définitivement terme à l'expérience ${ }^{56}$. Et c'est ainsi que s'achève, sur un désaveu, un épisode de la vie de Chen 
Yun durant lequel il s'est montré tour à tour conservateur (en refusant des changements trop brusques), pragmatique (en essayant de composer avec la réalité politico-économique qui s'imposait à lui), et réformiste (en proposant des aménagements qui pouvaient apparaître audacieux dans le contexte). La période qui s'ouvre à lui va être publiquement beaucoup moins active : c'est dans une semi-disgrâce qu'il continue de conseiller les responsables de l'économie entre 1957 et 1965.

Thierry Pairault est Chargé de recherches au CNRS

\section{TABLEAUX}

Tableau 1: Indices des prix de gros en 1950 (base 100 : décembre 1949)

janvier

126,6

juillet

166,8

février

203,3

août

173,6

mars

226,3

septembre

177,8

avril

169,9

octobre

185,8

mai

156,7

novembre

193,3

juin

155,8

décembre

193,2

Source : JJYJ 1978, p. 63

Tableau 2: Répartition des entreprises industrielles

(en \% de la production industrielle totale d'une année)

secteur socia-

liste

1949

1950

1951

1952 secteur capi-

taliste

sous contrôle

gestion sous

mixte contrat secteur capi-

taliste

libre

$\begin{array}{rrr}2,0 & 7,5 & 55,8 \\ 2,9 & 14,9 & 36,9 \\ 4,0 & 21,4 & 28,7 \\ 5,0 & 21,9 & 17,1\end{array}$

Source: $H C$, p. 20. 
Tableau 3 : Composition de la Commission d'État au plan (1952)

CEP N.E. ECO destin observations

D $\quad \mathbf{P}$

\begin{tabular}{|c|c|c|c|c|c|c|}
\hline $\begin{array}{l}\text { Gao Gang } \\
\text { Li Fuchun }\end{array}$ & $\begin{array}{l}\mathrm{P} \\
\mathrm{V}\end{array}$ & $\begin{array}{l}x \\
x\end{array}$ & $\mathbf{E}$ & 69 & 55 & $\begin{array}{l}\text { "suicidé» } \\
\text { avec Gao Gang au Xibei }\end{array}$ \\
\hline Deng Zihui & V & & & 57 & 65 & \\
\hline Jia Tuofu & & & $\mathrm{E}$ & & 60 & $\begin{array}{l}\text { "effet" Peng Dehuai; } \\
\text { avec Gao Gang au Xibei }\end{array}$ \\
\hline $\begin{array}{l}\text { Ma Hong } \\
\text { An Zhiwen }\end{array}$ & $\begin{array}{l}\mathbf{S} \\
\mathbf{m}\end{array}$ & $\begin{array}{l}x \\
x\end{array}$ & $\begin{array}{l}\mathbf{E} \\
\mathbf{E}\end{array}$ & 55 & 66 & actif après le Grand bond \\
\hline Bo Yibo & m & & $\stackrel{\mathbf{L}}{\mathbf{E}}$ & & $\begin{array}{l}00 \\
66\end{array}$ & \\
\hline $\begin{array}{l}\text { Chen Yun } \\
\text { Deng }\end{array}$ & $\mathbf{m}$ & $\mathbf{x}$ & $\mathbf{E}$ & 57 & $66 ?$ & \\
\hline Xiaoping & $\mathbf{m}$ & & & & 66 & \\
\hline Huang & & & & & & \\
\hline $\begin{array}{l}\text { Kecheng } \\
\text { Lin Biao }\end{array}$ & $\mathrm{m}$ & $x$ & & & 59 & $\begin{array}{l}\text { "effet" Peng Dehuai } \\
\text { fuite explosive }\end{array}$ \\
\hline Liu Lantao & m & $\mathbf{x}$ & & & 66 & \\
\hline Peng Dehuai & $\mathbf{m}$ & $\mathbf{x}$ & & & 59 & \\
\hline Peng Zhen & $\mathbf{m}$ & $\mathbf{x}$ & & & 66 & $\begin{array}{l}\text { frictions avec Gao Gang en } \\
46-48 \text {, réglées par Mao en } \\
\text { faveur de Gao }\end{array}$ \\
\hline ao Sh & m & & & & 55 & \\
\hline Xi 2 & $\mathbf{m}$ & & & 62 & 66 & $\begin{array}{l}62 \text { : "effet" Peng Dehuai; } \\
\text { avec Gao Gang au Xibei }\end{array}$ \\
\hline $\begin{array}{l}\text { Xue Mugiao } \\
\text { Zhang Xi }\end{array}$ & $\mathbf{m}$ & & $\mathbf{E}$ & & 66 & décès naturel en 59 \\
\hline
\end{tabular}

Abréviations :

CEP : Commission d'État au plan.

$P$ : président; $\mathbf{V}$ : vice-président.

$\mathrm{S}$ : secrétaire; $\mathrm{m}$ : membre

N.E.: si «x» a exercé des responsabilités dans la région Nord-Est.

ECO : si «E» doit être considéré comme un économiste.

$D$ : disgrâce; $P$ : purge. (Le chiffre donne la date :19xx). 


\section{NOTES}

Abréviations :

CB : Current background

CWa : Chen Yun wenxuan, 1949-1956

CWb : Chen Yun wenxuan, 1956-1985

Dashiji : Zhonghua renmin gongheguo jingji dashiji

Fagui : Zhonghua renmin gongheguo fagui huibian

Faling : Zhongyang renmin zhengfu faling huibian

$H C$ : Xin Zhongguo shangye shigao

M5 : Mao Zedong xuanji, vol. 5

RMSC : Renmin shouce

1. $C W a$, pp. 126-139.

2. Pour un exposé clair de l'alliance entre ouvriers et paysans proposée par Boukharine, voir Cohen 1979, pp. 201-235.

3. CWa, pp. 117-120.

4. Voir par exemple le texte des Gardes rouges reproduit dans Zhanwang, 411 (1979), pp. 25-27.

5. CWa, pp. 14-15.

6. La décision et les textes qui l'accompagnent sont dans Faling, vol. 1, pp. 239 sq.

7. Faling, vol. 1, pp. 388 sq.

8. Voir Meng 1951 , p. 366.

9. Cette reconstitution est déduite de $C W a$, pp. 1-110; Liu 1980, pp. 51-60; HC, pp. 8-14; RMSC 1951, pp. IX-14 à 26.

10. Su 1978, p. 61.

11. CWa, p. 93.

12. $C W a$, p. 106.

13. $H C$, p. 15.

14. CWa, pp. 167-181.

15. Faling, vol. 2, p. 199; Cihai, pp. 467-475.

16. Pour ces deux textes, voir Faling, vol. 2, pp. 419-425 et 533-535.

17. $H C$, p. 28.

18. Faling, vol. 3, pp. $41-43$.

19. Faling, vol. 2, pp. 145-147.

20. CWa, p. 133.

21. Nous excluons volontairement le cas de Peng Dehuai, ainsi que celui de Jia Tuofu, qui auraient défendu des options économiques proches de celles de Gao Gang. Si de telles accusations ont pu être reprises lors de la Révolution culturelle, elles datent de la fin du Grand Bond en avant, et non de l'affaire Gao Gang.

22. CWa, p. 45 et p. 347, n, 66.

23. Pour une présentation un tantinet hagiographique, voir ¿Chen Yun wenxuan, fudao jiangzuo, pp. 198 sq.

24. Voir le chapitre économique de Xinhua yuebao, 1959 (12), pp. 106 sq. 
25. Voir Guillermaz 1972, p. 109; Thornton 1973, pp. 230-234. Ce n'est pas parce que Gao Gang aurait eu des relations privilégiées avec les Soviétiques qu'il doit être considéré comme plus pro-soviétique que tel autre dirigeant chinois.

26. Notons qu'il a pu exister une certaine compétition entre Chen Yun et Gao Gang pour l'exercice des responsabilités économiques : avant 1949, en Mandchourie, le premier était président de la Commission pour le développement économique, président du Bureau des finances et président du Bureau des transports ferroviaires; le second président de la Commission économique et financière, en même temps que premier secrétaire. En outre, dans la hiérarchie gouvernementale après 1949, Chen Yun est subordonné à Gao Gang : en effet, le premier n'est que vice-premier ministre, alors que le second est vice-président du Gouvernement populaire central.

27. $H C$, pp. $37-38 ; C W a$, pp. $192-195 ; M 5$, pp. $90-97$.

28. Lieberthal 1976, pp. 59-60.

29. Voir Thornton 1973, pp. 230-232.

30. Voir les chapitres économiques de Xinhua yuebao, 1953 (5), pp. 137 sq.; (7), pp. 106 sq.; ainsi que le discours.de Jia Tuofu, (5), pp. 43-45.

31. Voir Gong 1985, pp. 76-75 (sic).

32. Ibid., pp. 226-227, permet d'établir que c'est bien $\mathrm{Li}$ Weihan, même si son rapport n'a jamais été publié (voir la table des matières dans $\mathrm{Li}$ 1982).

33. M5, pp. 90-97.

34. Texte in Faling, vol. 3, pp. 125-133.

35. Zhanwang, 409 (1979), p. 26; 411, par ex. p. 25.

36. CWa, pp. 196 sq.

37. Pour le développement qui suit, voir $X u$ et Chen 1984, pp. 259-260; Pei et Yang 1984, p. 85; $H C$, pp. 34-39; $C W a$, pp. 192201.

38. Voir $C W b$, pp. 205-206.

39. Pour une présentation plus complète, voir Guillermaz 1972, pp. 109-111; Lieberthal 1976, pp. 62-64; Rice 1974, pp. 128-132; China News Analysis, 80.

40. $C B, 290$.

41. $C W a$, p. 234.

42. Voir Zhanwang, 409 (1979), p. 26; 411, pp. 25-26; Xu 1974, pp. 194-196.

43. $C W a, p p$. 245-279.

44. In Ma 1980, pp. 167-217.

45. Voir Ma 1980, pp. 167-168, et Zhanwang, loc. cit.

46. Le texte se trouve dans Faling, vol. 5, pp. 81-85. Sur l'intervention de $\mathrm{Li}$ Weihan, voir Dashiji, p. 89; le texte de l'intervention se trouve dans $L i$ 1982, pp. 91-102.

47. Voir Deng et Ma 1985, p. 35.

48. Voir les tableaux 7, 8 et 10 in Aubert 1985. 
49. Faling, vol. 4, pp. 177-179.

50. Voir les éditoriaux du Dagongbao des 12 février, 11 mars et 28 mai 1955, et du Renmin ribao du 10 mars 1955.

51. Voir le discours de $\mathrm{Li}$ Weihan lors de la $3 e$ session de l'ANP in Li 1982, pp. 150-161.

52. $C W a$, pp. $307-326$.

53. Texte in RMSC1957, pp. 85-88.

54. Ma 1981, pp. 132-133.

55. Décret in Fagui, vol. 4, pp. 330-332.

56. Dagongbao, 28 janvier 1957 , p. 1.

\section{RÉFÉRENCES BIBLIOGRAPHIQUES}

\section{Textes de Chen Yun et hagiographies en langue chinoise}

Chen Yun tongzhi wengao xuanbian 1956-1962 (Euvres choisies du camarade Chen Yun, 1949-1962), Canton, Guangdong renmin chubanshe, 1981

Chen Yun wengao xuanbian 1949-1956 (Eurres choisies de Chen Yun, 1949-1956), Pékin, Renmin chubanshe, 1981.

*Chen Yun wenxuan fudao jiangzuo (Présentation des $\boldsymbol{E}_{u u r e s}$ choisies de Chen Yun), Pékin, Xinhua chubanshe, 1984.

Chen Yun wenxuan 1926-1949 (Euvres choisies de Chen Yun, 1926-1949), Pékin, Renmin chubanshe, 1984.

Chen Yun wenxuan 1949-1956 (Guvres choisies de Chen Yun, 1949-1956), Pékin, Renmin chubanshe, 1984.

Chen Yun wenxuan 1956-1985 (Euvres choisies de Chen Yun, 1956-1985), Pékin, Renmin chubanshe, 1986.

Deng Liqun, Xiang Chen Yun tongzhi xuexi zuo jingji gongzuo (Étudier comment le camarade Chen Yun accomplit le travail économique), Pékin, Zhongguo zhongyang dang xiao chubanshe, 1981.

Deng Liqun, Tantan jihua diaojie he shichang diaojie (De la régulation par le plan et la régulation par le marché), Pékin, Renmin chubanshe, 1979.

Guan Luojue, Chen Yun tongzhi de jingji sixiang (La pensée économique du camarade Chen Yun), Shanghai, Zhishi chubanshe, 1984.

He Jianzhang, "Wei renmin mo fuli shi shehuizhuyi jianshe de jiben fangzhen" (L'intérêt du peuple est le principe de base de la construction du socialisme), Jingii yanjiu, 1981 (11), pp. 23- 27.

Pei Yuanxiu, Yang Rongshen, Chen Yun caizheng lunzhu qiantan (Présentation des travaux de Chen Yun sur les finances publiques), Pékin, Xinhua chubanshe, 1984. 


\section{Autres sources}

Aubert, Claude, Chine, le décollage alimentaire?, manuscrit, Paris, INRA, 1985.

Bianco, Lucien et Yves Chevrier (éds.), Dictionnaire biographique du mouvement ouvrier international. La Chine, Paris, Editions ouvrières/Presses de la FNSP, 1985.

Chen Zengji, *Cong woguo lishi jingyan kan guomin jingii zonghe pingheng zhong de jige wenti» (Quelques problèmes concernant l'équilibre global de l'économie nationale vus à partir de l'expérience historique chinoise), in Zhongguo caizheng wenti, Tianjin, Tianjin kexue jishu chubanshe, 1981, pp. 281-293.

Cihai. Jingji fence (Annexe économique au dictionnaire Cihai), Shanghai, Shanghai cishu chubanshe, 1982.

Cohen, Stephen, Nicolas Boukharine. La vie d'un bolchevik, Paris, Maspero, 1979. Unis.

Current background, Hong Kong, Consulat général des États-

Deng Liqun, Ma Hong et al. (eds.), Dangdai Zhongguo de jingji tizhi gaige (La réforme du système économique dans la Chine d'aujourd'hui), Pékin, Zhongguo shehui kexue chubanshe, 1984.

Deng Liqun, Ma Hong et al. (eds.), Dangdai Zhongguo de jingji guanli (La gestion économique dans la Chine d'aujourd'hui), Pékin, Zhongguo shehui kexue chubanshe, 1985.

Gong Yuzhi et al. (eds.), Guanyu jianguo yilai dang de ruogan lishi wenti de jueyi zhushi ben (xiuding) (Présentation de la décision sur quelques problèmes historiques concernant le Parti depuis la fondation de la RPC, éd. revue et augmentée), Pékin, Renmin chubanshe, 1985.

Guillermaz, Jacques, Le Parti communiste chinois au pouvoir, Paris, Payot, 1972.

Li Weihan, Tongyi zhanxian wenti yu minzu wenti (Problèmes du Front uni et problèmes des minorités nationales), Pékin, Renmin chubanshe, 1982.

Ma Yinchu, «Lianxi woguo shiji lai tantan zonghe pingheng lilun he an bili fazhan guilu (Discutons de la loi du développement proportionné et de la théorie de l'équilibre global en fonction de la réalité chinoise), in $M a$ Yinchu jingji lunwen xuanji, Pékin, Beijing daxue renmin chubanshe, 1981.

Ma Yinchu, "Woguo zibenzhuyi gongye de shehuizhuyi gaizao» (La transformation socialiste de l'industrie capitaliste en Chine), in $\mathrm{Ma}$, Wode jingji lilun, zhexue sixiang he zhengzhi lichang (Mes théories économiques, ma pensée philosophique et ma position politique), Pékin, Zhongguo caizheng jingji chubanshe, 1980, pp. 167-217.

Meng Xianzhang, Zhongguo jindai jingji shi jiaocheng (Manuel d'histoire économique de la Chine moderne), Shanghai, Zhonghua shuju, 1951. 
Lardy, Nicholas et Kenneth Lieberthal (eds.), Chen Yun's strategy for China's development : a non-Maoist alternative, New York, Sharpe, 1983.

Lieberthal, Kenneth, $A$ research guide to central party and government meetings in China, 1949-1975, New York, LASP, 1976.

Liu Hongru, Shehuizhuyi huobi yu yinhang wenti (Le problème de la monnaie et des banques sous le socialisme), Pékin, Zhongguo caizheng jingii chubanshe, 1980.

MacFarquhar, Roderick, The origins of the Cultural Revolution, 2 vol., Oxford, Oxford University Press, 1974 et 1983.

Mao Zedong xuanji (Euvres choisies de Mao Zedong), vol. 5, Pékin, Renmin chubanshe, 1977.

Rice, Edward, Mao's way, Berkeley, University of California Press, 1974.

Su Xing, "Woguo caizheng jingji gongzuo de lishi zhuanzhedian" (Un tournant historique dans le travail économique et financier de notre pays), Jingji yanjiu, 1978 (3), pp. 55-64.

Sun Jian, Zhonghua renmin gongheguo jingii shigao 1946-1957 (Histoire économique de la RPC, 1949-1957), Changchun, Jilin renmin chubanshe, 1980.

Tang, Peter S. H., Communist China today: domestic and foreign policies, New York, Praeger, 1957.

Thornton, Richard C., China : the struggle for power, 1917. 1972, Bloomington, Indiana University Press, 1973.

Xin Zhongguo shangye shigao (Histoire du commerce dans la nouvelle Chine), Pékin, Zhongguo caizheng jingii chubanshe, 1984.

$\mathrm{Xu}$ Deming [Hsu Tak-ming], Wenge hou de Zhonggong jingji (L'économie chinoise après la Révolution culturelle), Hong Kong, Union Research Institute, 1974.

$\mathrm{Xu}$ Yi et Chen Baosen, Caizhengxue (Les finances publiques), Pékin, Zhongguo caizheng chubanshe, 1984.

Xue Muqiao, Su Xing et al., Zhongguo guomin jingji de shehuizhuyi gaizao (La transformation socialiste de l'économie nationale en Chine), Pékin, Renmin chubanshe, 1959, 1978.

Zhonggong renming lu (Biographies de personnalités du PCC), Taipei, Guoli zhengzhi daxue guoji guanxi yanjiu zhongxin, 1978.

Zhonghua renmin gongheguo fagui huibian (Recueil des lois de la RPC), Pékin, Falü chubanshe, 1981-1982.

Zhonghua renmin gongheguo jingji dashiji (1949 nian 10 yue1984 nian 9 yue) (Répertoire des grands faits économiques en RPC, oct. 1949-sept. 1984), Pékin, Beijing chubanshe, 1985.

Zhongyang renmin zhengfu faling huibian (Recueil des lois du gouvernement populaire central), Pékin, Falü chubanshe, 1982. 